MATHEMATICS OF COMPUTATION

Volume 78, Number 268, October 2009, Pages 1975-1995

S 0025-5718(09)02234-0

Article electronically published on February 23, 2009

\title{
DISCONTINUOUS GALERKIN METHOD FOR AN EVOLUTION EQUATION WITH A MEMORY TERM OF POSITIVE TYPE
}

\author{
KASSEM MUSTAPHA AND WILLIAM MCLEAN
}

\begin{abstract}
We consider an initial value problem for a class of evolution equations incorporating a memory term with a weakly singular kernel bounded by $C(t-s)^{\alpha-1}$, where $0<\alpha<1$. For the time discretization we apply the discontinuous Galerkin method using piecewise polynomials of degree at most $q-1$, for $q=1$ or 2 . For the space discretization we use continuous piecewise-linear finite elements. The discrete solution satisfies an error bound of order $k^{q}+h^{2} \ell(k)$, where $k$ and $h$ are the mesh sizes in time and space, respectively, and $\ell(k)=\max \left(1, \log k^{-1}\right)$. In the case $q=2$, we prove a higher convergence rate of order $k^{3}+h^{2} \ell(k)$ at the nodes of the time mesh. Typically, the partial derivatives of the exact solution are singular at $t=0$, necessitating the use of non-uniform time steps. We compare our theoretical error bounds with the results of numerical computations.
\end{abstract}

\section{INTRODUCTION}

We study the discretization in time and space of an initial value problem [1, 3, 9, 11, 12, 18, 16,

$$
\frac{\partial u}{\partial t}+\mathcal{B} A u=f(t) \quad \text { for } 0<t<T, \quad \text { with } u(0)=u_{0},
$$

where $\mathcal{B}$ denotes a Volterra integral operator

$$
\mathcal{B} v(t)=\int_{0}^{t} \beta(t, s) v(s) d s
$$

and where $A$ is a selfadjoint linear operator with domain $D(A)$ in a real Hilbert space $\mathbb{H}$. We assume that $A$ has a complete eigensystem $\left\{\lambda_{m}, \phi_{m}\right\}_{m=1}^{\infty}$ such that $0 \leq \lambda_{1} \leq \lambda_{2} \leq \cdots$ and $\lambda_{m} \rightarrow \infty$ as $m \rightarrow \infty$. Thus, $A$ is positive semidefinite. The solution $u$ and source term $f$ take values in $\mathbb{H}$, and the initial data $u_{0}$ is an element of $\mathbb{H}$. We let $\langle u, v\rangle$ denote the inner product of $u$ and $v$ in $\mathbb{H}$, and define the bilinear form

$$
A(u, v)=\langle A u, v\rangle=\sum_{m=1}^{\infty} \lambda_{m}\left\langle u, \phi_{m}\right\rangle\left\langle\phi_{m}, v\right\rangle \quad \text { for } u, v \in D\left(A^{1 / 2}\right) .
$$

Received by the editor October 16, 2007 and, in revised form, October 9, 2008.

2000 Mathematics Subject Classification. Primary 26A33, 45J05, 65M12, 65M15, 65M60.

Key words and phrases. Memory term, discontinuous Galerkin method, a priori error estimates, non-uniform time steps, finite element method.

Support of the KFUPM is gratefully acknowledged.

(C)2009 American Mathematical Society Reverts to public domain 28 years from publication 
Concretely, one may take $\mathbb{H}=L_{2}(\Omega)$ for a bounded, Lipschitz domain $\Omega \subseteq \mathbb{R}^{d}$ and $A=-\nabla^{2}$ subject to homogeneous Dirichlet or Neumann boundary conditions. In this case, $u=u(x, t), f=f(x, t)$ and $u_{0}=u_{0}(x)$ for $x \in \Omega$ and $t>0$, with $A(u, v)=\int_{\Omega} \nabla u \cdot \nabla v d x$.

Throughout the paper, we assume the kernel $\beta$ to be real-valued and strictly positive definite, that is,

$$
\int_{0}^{T} v(t) \int_{0}^{t} \beta(t, s) v(s) d s d t \geq 0 \quad \text { for all } v \in L_{\infty}([0, T], \mathbb{R}),
$$

with equality if and only if $v$ is zero almost everywhere on $[0, T]$. In addition, $\beta$ may be at worst weakly singular, that is,

$$
|\beta(t, s)| \leq C(t-s)^{\alpha-1} \quad \text { for } 0<s<t<\infty \text {, with } 0<\alpha \leq 1,
$$

and we assume for simplicity that $\beta(t, s)$ is continuous for $t \neq s$. Of particular interest is the choice

$$
\beta(t, s)=\frac{(t-s)^{\alpha-1}}{\Gamma(\alpha)},
$$

which makes $\mathcal{B}$ the Riemann-Liouville fractional integration operator of order $\alpha$ and means that the evolution equation in (1.1) is a fractional wave equation [18.

A standard approach 9, 12, 16, to the time discretization of (1.1) uses a combination of finite differences and quadrature. If the kernel $\beta(t, s)$ depends only on the difference $t-s$, then convolution quadrature [3, 10] is a natural choice, allowing the use of fast summation techniques [17. Another approach [7, 8, 13, 14, again suitable for a convolution kernel, achieves spectral accuracy via numerical inversion of the Laplace transform of the solution. In the present work, we instead apply the discontinuous Galerkin method using piecewise polynomials of degree at most $q-1$, for $q=1$ or 2 .

Since their inception in the early 1970s, discontinuous Galerkin methods have found numerous applications [2], including for the time discretization of parabolic problems [5]. Their advantages include excellent stability properties even for highly non-uniform meshes and suitability for adaptive refinement based on a posteriori error estimates 4 to handle problems with low regularity. McLean, Thomée and Wahlbin [15] proved a priori error estimates for a piecewise-constant $(q=1)$ discontinuous Galerkin method applied to (1.1), although they formulated the method as a generalised backward-Euler scheme; see (1.7) below. Adolfsson, Enelund and Larsson [1] subsequently proved a posteriori error estimates for the same piecewiseconstant discontinuous Galerkin method, leading to adaptive control of the step size. They also incorporated the use of sparse quadrature to reduce the computational cost of the algorithm. Neither [15] nor [1] considered the spatial discretization of (1.1).

Here, we focus on the piecewise-linear case $(q=2)$ and consider only a priori error bounds.

To set up the time discretization, we begin with a (possibly non-uniform) partition of the interval $[0, T]$,

$$
0=t_{0}<t_{1}<\cdots<t_{N}=T \text { with } I_{n}=\left(t_{n-1}, t_{n}\right] .
$$

We denote the $n$th step-size by $k_{n}=t_{n}-t_{n-1}$ and the maximum step-size by $k=\max _{1 \leq n \leq N} k_{n}$. For $q \geq 1$, we let $\mathbb{P}_{q}$ denote the space of polynomials of degree strictly less than $q$ with coefficients in $D\left(A^{1 / 2}\right)$. For $q=1$ or 2 , our trial space $\mathcal{W}_{q}$ 
is the set of functions $U:[0, T] \rightarrow D\left(A^{1 / 2}\right)$ such that $\left.U\right|_{I_{n}} \in \mathbb{P}_{q}$ for $1 \leq n \leq N$. We follow the usual convention that a function $U \in \mathcal{W}_{q}$ is left-continuous at each time level $t_{n}$, writing

$$
U^{n}=U\left(t_{n}\right)=U\left(t_{n}^{-}\right), \quad U_{+}^{n}=U\left(t_{n}^{+}\right), \quad[U]^{n}=U_{+}^{n}-U^{n} .
$$

For any continuous test function $v:[0, T] \rightarrow D\left(A^{1 / 2}\right)$, the solution $u$ of (1.1) satisfies

$$
\int_{t_{n-1}}^{t_{n}}\left[\left\langle u^{\prime}(t), v(t)\right\rangle+A(\mathcal{B} u(t), v(t))\right] d t=\int_{t_{n-1}}^{t_{n}}\langle f(t), v(t)\rangle d t .
$$

By comparison, given $U(t)$ for $0 \leq t \leq t_{n-1}$, the discontinuous Galerkin method determines $U \in \mathcal{W}_{q}$ on $I_{n}$ by requiring that

$$
\begin{aligned}
\left\langle U_{+}^{n-1}, X_{+}^{n-1}\right\rangle+\int_{t_{n-1}}^{t_{n}}\left[\left\langle U^{\prime}(t), X(t)\right\rangle\right. & +A(\mathcal{B} U(t), X(t))] d t \\
& =\left\langle U^{n-1}, X_{+}^{n-1}\right\rangle+\int_{t_{n-1}}^{t_{n}}\langle f(t), X(t)\rangle d t
\end{aligned}
$$

for all $X \in \mathbb{P}_{q}\left(I_{n}\right)$. This time-stepping procedure starts from $U^{0} \approx u_{0}$, and after $N$ steps yields the numerical solution $U(t)$ for $0 \leq t \leq t_{N}$.

For the piecewise-constant case $q=1$, since $U^{\prime}(t)=0$ and $U(t)=U^{n}=U_{+}^{n-1}$ for $t \in I_{n}$, the discontinuous Galerkin method (1.6) amounts to a generalized backward-Euler scheme

$$
\frac{U^{n}-U^{n-1}}{k_{n}}+\mathcal{B}^{n}(A U)=\bar{f}^{n}
$$

where

$$
\begin{gathered}
\mathcal{B}^{n}(A U)=\frac{1}{k_{n}} \int_{t_{n-1}}^{t_{n}} \int_{0}^{t} \beta(t, s) A U(s) d s d t=\sum_{j=1}^{n} \omega_{n j} A U^{j} k_{j}, \\
\bar{f}^{n}=\frac{1}{k_{n}} \int_{t_{n-1}}^{t_{n}} f(t) d t, \quad \omega_{n j}=\frac{1}{k_{n} k_{j}} \int_{t_{n-1}}^{t_{n}} \int_{t_{j-1}}^{\min \left(t, t_{j}\right)} \beta(t, s) d s d t .
\end{gathered}
$$

Thus, at each time step we must solve an "elliptic" problem

$$
U^{n}+k_{n}^{2} \omega_{n n} A U^{n}=U^{n-1}+k_{n} \bar{f}_{n}-k_{n} \sum_{j=1}^{n-1} \omega_{n j} A U^{j} k_{j} .
$$

For the piecewise-linear case $q=2$, we define

$$
\psi_{n}^{1}(t)=\frac{t_{n}-t}{k_{n}} \quad \text { and } \quad \psi_{n}^{2}(t)=\frac{t-t_{n-1}}{k_{n}}
$$

and use the representation

$$
U(t)=U_{+}^{n-1} \psi_{n}^{1}(t)+U^{n} \psi_{n}^{2}(t) \quad \text { for } t \in I_{n} .
$$


By choosing $X(t)=\psi_{n}^{p}(t) V$ in (1.6) for $p \in\{1,2\}$ and a vector $V \in \mathbb{H}$ (independent of $t$ ), we arrive at the $2 \times 2$ system

$$
\begin{aligned}
\left(\frac{1}{2}+\omega_{n n}^{11} A\right) U_{+}^{n-1}+\left(\frac{1}{2}+\omega_{n n}^{12} A\right) U^{n} & =U^{n-1}+f^{n 1}-\sum_{j=1}^{n-1}\left(\omega_{n j}^{11} A U_{+}^{j-1}+\omega_{n j}^{12} A U^{j}\right), \\
\left(-\frac{1}{2}+\omega_{n n}^{21} A\right) U_{+}^{n-1}+\left(\frac{1}{2}+\omega_{n n}^{22} A\right) U^{n} & =f^{n 2}-\sum_{j=1}^{n-1}\left(\omega_{n j}^{21} U_{+}^{j-1}+\omega_{n j}^{22} U^{j}\right),
\end{aligned}
$$

where

$$
\omega_{n j}^{p r}=\int_{t_{n-1}}^{t_{n}} \psi_{n}^{p}(t) \int_{t_{j-1}}^{\min \left(t, t_{j}\right)} \beta(t, s) \psi_{j}^{r}(s) d s d t \quad \text { and } \quad f^{n p}=\int_{t_{n-1}}^{t_{n}} f(t) \psi_{n}^{p}(t) d t .
$$

For a general $q$, we would have to solve a $q \times q$ system.

The regularity results in [3, 11, 12] show, for the specific weakly singular kernel (1.3) and under reasonable assumptions on the data $u_{0}$ and $f(t)$, that there exist constants $\sigma$ and $M$, with $0<\sigma \leq 1$, such that the exact solution of (1.1) satisfies

$$
t\left\|A u^{\prime}(t)\right\|+t^{2}\left\|A u^{\prime \prime}(t)\right\| \leq M t^{\sigma-1} \text { for } 0<t \leq T
$$

and

$$
\left\|u^{\prime}(t)\right\|+t\left\|u^{\prime \prime}(t)\right\| \leq M t^{\sigma-1} \quad \text { for } 0<t \leq T .
$$

This singular behaviour as $t \rightarrow 0^{+}$may lead to suboptimal convergence rates if we work with quasi-uniform time meshes. We therefore assume that, for a fixed $\gamma \geq 1$,

$$
k_{n} \leq C k t_{n}^{1-1 / \gamma} \text { and } t_{n} \leq C t_{n-1} \quad \text { for } 2 \leq n \leq N,
$$

with

$$
c k^{\gamma} \leq k_{1} \leq C k^{\gamma}
$$

For instance, we may choose

$$
t_{n}=(n / N)^{\gamma} T \text { for } 0 \leq n \leq N .
$$

We show in Theorem 3.2 that the error $\|U(t)-u(t)\|$ is of order $k^{q}$, uniformly for $0 \leq t \leq T$, provided $\gamma>q / \sigma$ and the initial data satisfy $\left\|U^{0}-u_{0}\right\|=O\left(k^{q}\right)$. However, for a quasi-uniform mesh our bound yields a poorer convergence rate of order $k^{\sigma}$.

In the piecewise-linear case $q=2$, faster convergence than $O\left(k^{2}\right)$ is possible at the nodal points $t_{n}$. The nodal error $\left\|U^{n}-u\left(t_{n}\right)\right\|$ is $O\left(k^{3}\right)$ if $\beta$ and $u$ are smooth, and the same result holds for the weakly-singular kernel (1.3) and for $u$ satisfying (1.9), provided the mesh grading parameter $\gamma>3 /(\sigma+\alpha)$; see Corollary 4.2. Compare these results with those of Eriksson, Johnson and Thomée [5] for the classical parabolic problem that arises if one takes $\mathcal{B} v=v$ in (1.1): the error is then $O\left(k^{q}\right)$ everywhere on $[0, T]$ and is $O\left(k^{2 q-1}\right)$ at the nodes, for a general $q \geq 1$. In related work, Larsson, Thomée and Wahlbin [6] proved the same convergence rates for a parabolic integrodifferential equation of the form $\partial u / \partial t+A u+$ memory term $=f(t)$, using discontinuous Galerkin methods with $q=1$ or 2 . 
In the concrete setting where $\mathbb{H}=L_{2}(\Omega)$ and $A=-\nabla^{2}$, we discretize in space using standard, continuous, piecewise-linear finite elements on a quasi-uniform partition of the domain $\Omega$ to obtain a numerical solution $U_{h}$. Under the additional regularity assumptions

$$
\left\|u_{0}\right\|_{2} \leq M \quad \text { and } \quad\|u(t)\|_{2}+t\left\|u^{\prime}(t)\right\|_{2} \leq M \quad \text { for } 0<t \leq T,
$$

where $\|v\|_{2}=\|v\|_{H^{2}(\Omega)}$, we show in Theorem 5.2 that, with

$$
\ell(k)=\max \left(1, \log k^{-1}\right),
$$

the error $\left\|U_{h}(t)-u(t)\right\|$ is of order $k^{q}+h^{2} \ell(k)$, uniformly for $0 \leq t \leq T$, provided $\gamma>q / \sigma$. Our final result, Corollary [5.4 establishes an improved error bound of order $k^{3}+h^{2} \ell(k)$ for $U_{h}$ at the nodes $t=t_{n}$, when $q=2$ and $\beta$ is as in (1.3).

The concluding section of the paper presents the results of some numerical computations that confirm our theoretical error bounds.

\section{StabiLity}

An energy argument based on the positive-semidefiniteness of $\mathcal{B}$ and $A$ implies the existence and uniqueness of a mild solution $u \in C([0, T] ; \mathbb{H})$ to the continuous problem (1.1), and yields a stability estimate [12,

$$
\|u(t)\| \leq\left\|u_{0}\right\|+2 \int_{0}^{t}\|f(s)\| d s .
$$

To state an analogous result for the discrete problem (1.6), we introduce the notation

$$
\|U\|_{J}=\sup _{t \in J}\|U(t)\|
$$

for any subinterval $J \subseteq[0, T]$, and put $J_{n}=\left(0, t_{n}\right]=\bigcup_{j=1}^{n} I_{j}$. Note that the proof makes no assumptions on the mesh (1.4).

Theorem 2.1. Let $q \in\{1,2\}$. Given $U^{0} \in \mathbb{H}$ and $f \in L_{1}((0, T) ; \mathbb{H})$, there exists a unique $U \in \mathcal{W}_{q}$ satisfying (1.6) for $n=1,2, \ldots, N$. Furthermore, $U(t) \in D(A)$ for $t>0$ and, with $C_{1}=2$ and $C_{2}=8$,

$$
\|U\|_{J_{n}} \leq C_{q}\left(\left\|U^{0}\right\|+\int_{0}^{t_{n}}\|f(t)\| d t\right) \quad \text { for } n \geq 1 .
$$

Proof. Recall the notation (1.5) and assume for the moment that $U$ exists. By choosing $X=U$ in (1.6) and using $\left\langle U^{\prime}(t), U(t)\right\rangle=(d / d t)\|U(t)\|^{2} / 2$, we obtain

$$
\begin{aligned}
\frac{1}{2}\left(\left\|U^{j}\right\|^{2}+\left\|U_{+}^{j-1}\right\|^{2}\right)+\int_{t_{j-1}}^{t_{j}} A(\mathcal{B} U(t), U(t)) d t & \\
= & \left\langle U^{j-1}, U_{+}^{j-1}\right\rangle+\int_{t_{j-1}}^{t_{j}}\langle f(t), U(t)\rangle d t .
\end{aligned}
$$

Since (1.2) implies

$$
\begin{aligned}
\sum_{j=1}^{n} \int_{t_{j-1}}^{t_{j}} A(\mathcal{B} U(t), U(t)) d t & =\int_{0}^{t_{n}} \int_{0}^{t} \beta(t, s) A(U(s), U(t)) d s d t \\
= & \sum_{m=1}^{\infty} \lambda_{m} \int_{0}^{t_{n}} \int_{0}^{t} \beta(t, s)\left\langle U(s), \phi_{m}\right\rangle d s\left\langle\phi_{m}, U(t)\right\rangle d t \geq 0
\end{aligned}
$$


we see that

$$
\sum_{j=1}^{n}\left(\left\|U^{j}\right\|^{2}+\left\|U_{+}^{j-1}\right\|^{2}\right) \leq 2 \sum_{j=1}^{n}\left\langle U^{j-1}, U_{+}^{j-1}\right\rangle+2 \int_{0}^{t_{n}}\langle f(t), U(t)\rangle d t,
$$

SO

$$
\begin{aligned}
\left\|U^{n}\right\|^{2}+\left\|U_{+}^{0}\right\|^{2}+\sum_{j=1}^{n-1}\left(\left\|U^{j}\right\|^{2}-2\left\langle U^{j}, U_{+}^{j}\right\rangle\right. & \left.+\left\|U_{+}^{j}\right\|^{2}\right) \\
& \leq 2\left\langle U^{0}, U_{+}^{0}\right\rangle+2 \int_{0}^{t_{n}}\langle f(t), U(t)\rangle d t
\end{aligned}
$$

and hence

$$
\left\|U^{n}\right\|^{2}+\left\|U_{+}^{0}\right\|^{2}+\sum_{j=1}^{n-1}\left\|[U]^{j}\right\|^{2} \leq 2\left(\left\|U^{0}\right\|\left\|U_{+}^{0}\right\|+\int_{0}^{t_{n}}\|f(t)\|\|U(t)\| d t\right) .
$$

Let $q=2$. Since $\|U\|_{I_{n}}=\max \left(\left\|U^{n}\right\|,\left\|U_{+}^{n-1}\right\|\right)$ we have

$$
\|U\|_{I_{1}}^{2} \leq\left\|U^{1}\right\|^{2}+\left\|U_{+}^{0}\right\|^{2} \leq 2\left(\left\|U^{0}\right\|\left\|U_{+}^{0}\right\|+\int_{0}^{t_{1}}\|f(t)\|\|U(t)\| d t\right),
$$

and, for $n \geq 2$,

$$
\begin{aligned}
\|U\|_{I_{n}}^{2} & =\max \left(\left\|U^{n}\right\|^{2},\left\|U_{+}^{n-1}\right\|^{2}\right)=\max \left(\left\|U^{n}\right\|^{2},\left\|U^{n-1}+[U]^{n}\right\|^{2}\right) \\
& \leq \max \left(\left\|U^{n}\right\|^{2}, 2\left\|U^{n-1}\right\|^{2}+2\left\|[U]^{n-1}\right\|^{2}\right) \\
& \leq 8\left(\left\|U^{0}\right\|\left\|U_{+}^{0}\right\|+\int_{0}^{t_{n}}\|f(t)\|\|U(t)\| d t\right) .
\end{aligned}
$$

Thus, putting $j_{n}=\arg \max _{1 \leq j \leq n}\|U\|_{I_{j}}$, the desired bound follows at once from

$$
\|U\|_{J_{n}}^{2}=\|U\|_{I_{j_{n}}}^{2} \leq 8\|U\|_{J_{n}}\left(\left\|U^{0}\right\|+\int_{0}^{t_{j_{n}}}\|f(t)\| d t\right)
$$

and we see that $U$ is unique.

When $\mathbb{H}$ is finite dimensional, the existence of $U$ follows from uniqueness because the square linear system (1.8) must be uniquely solvable. When $\mathbb{H}$ is infinite dimensional, we can construct $U$ by expanding in the eigenfunctions of $A$, because the $2 \times 2$ matrix

$$
\left[\begin{array}{cc}
\left(\frac{1}{2}+\omega_{n n}^{11} \lambda\right) & \left(\frac{1}{2}+\omega_{n n}^{12} \lambda\right) \\
\left(-\frac{1}{2}+\omega_{n n}^{21} \lambda\right) & \left(\frac{1}{2}+\omega_{n n}^{22} \lambda\right)
\end{array}\right]
$$

is non-singular for all $\lambda \geq 0$. Moreover, defining $V(t)=0$ for $t \notin I_{n}$, we see that the quadratic form

$$
\left[\begin{array}{ll}
V_{+}^{n-1} & V^{n}
\end{array}\right]\left[\begin{array}{cc}
\omega_{n n}^{11} & \omega_{n n}^{12} \\
\omega_{n n}^{21} & \omega_{n n}^{22}
\end{array}\right]\left[\begin{array}{c}
V_{+}^{n-1} \\
V^{n}
\end{array}\right]=\int_{0}^{T} V(t) \mathcal{B} V(t) d t
$$

is strictly positive-definite, and so the determinant of (2.2) is bounded below by $c \lambda^{2}$ for $\lambda$ sufficiently large. Thus, Cramer's rule shows that the norm of the inverse matrix is $O\left(\lambda^{-1}\right)$ as $\lambda \rightarrow \infty$, and a simple inductive argument gives $U_{+}^{n-1}, U^{n} \in$ $D(A)$ for $1 \leq n \leq N$, implying that $U(t) \in D(A)$ for $0<t \leq T$.

For $q=1$, we have $\|U\|_{I_{n}}=\left\|U^{n}\right\|$, so the stability bound with $C_{1}=2$ follows at once from (2.1), and $U^{n} \in D(A)$ follows from $\left(1+\omega_{n n} \lambda\right)^{-1}=O\left(\lambda^{-1}\right)$. 
The proof above also yields a bound for the jumps in the numerical solution.

Corollary 2.2. With $C_{1}=4$ and $C_{2}=16$,

$$
\sum_{j=1}^{n-1}\left\|[U]^{j}\right\|^{2} \leq C_{q}\left(\left\|U^{0}\right\|+\int_{0}^{t_{n}}\|f(t)\| d t\right)^{2} .
$$

Proof. Apply (2.1).

\section{ERROR FROM THE TIME DISCRETIZATION}

For our error analysis, we reformulate the discontinuous Galerkin method in terms of a global bilinear form

$$
\begin{aligned}
G_{N}(U, X)=\left\langle U_{+}^{0}, X_{+}^{0}\right\rangle+ & \sum_{n=1}^{N-1}\left\langle[U]^{n}, X_{+}^{n}\right\rangle \\
& +\sum_{n=1}^{N} \int_{t_{n-1}}^{t_{n}}\left[\left\langle U^{\prime}(t), X(t)\right\rangle+A(\mathcal{B} U(t), X(t))\right] d t .
\end{aligned}
$$

Summing the equations (1.6) gives

$$
G_{N}(U, X)=\left\langle U^{0}, X_{+}^{0}\right\rangle+\int_{0}^{t_{N}}\langle f(t), X(t)\rangle d t \quad \text { for all } X \in \mathcal{W}_{q}
$$

and conversely, by choosing $X$ to be identically zero outside $I_{n}$, we see that if $U \in$ $\mathcal{W}_{q}$ satisfies (3.2), then (1.6) holds for each $n$. Since the exact solution $u$ has no jumps,

and thus

$$
G_{N}(u, X)=\left\langle u_{0}, X_{+}^{0}\right\rangle+\int_{0}^{t_{N}}\langle f(t), X(t)\rangle d t
$$

$$
G_{N}(U-u, X)=\left\langle U^{0}-u_{0}, X_{+}^{0}\right\rangle \text { for all } X \in \mathcal{W}_{q} .
$$

Integration by parts yields an alternative expression for the bilinear form (3.1),

$$
\begin{aligned}
G_{N}(U, X)=\left\langle U^{N}, X^{N}\right\rangle & -\sum_{n=1}^{N-1}\left\langle U^{n},[X]^{n}\right\rangle \\
& +\sum_{n=1}^{N} \int_{t_{n-1}}^{t_{n}}\left[-\left\langle U(t), X^{\prime}(t)\right\rangle+A(\mathcal{B} U(t), X(t))\right] d t .
\end{aligned}
$$

For any continuous function $u:[0, T] \rightarrow \mathbb{H}$ we define a piecewise-constant interpolant $\Pi u:[0, T] \rightarrow \mathcal{W}_{1}$ by putting

$$
\Pi u(t)=u\left(t_{n}\right) \quad \text { for } t \in I_{n}, \quad \text { with } \Pi u(0)=u(0),
$$

and observe that if $u^{\prime}$ is integrable, then the interpolation error has the representation

$$
\Pi u(t)-u(t)=\int_{t}^{t_{n}} u^{\prime}(s) d s \quad \text { for } t \in I_{n} .
$$

In the piecewise-linear case, we define $\Pi u:[0, T] \rightarrow \mathcal{W}_{2}$ by requiring that

$$
\Pi u\left(t_{n}\right)=u\left(t_{n}\right) \quad \text { and } \quad \int_{t_{n-1}}^{t_{n}}[u(t)-\Pi u(t)] d t=0,
$$


with $\Pi u(0)=u(0)$. Explicitly, we find that

$$
\Pi u(t)=u\left(t_{n}\right)+\frac{u\left(t_{n}\right)-\bar{u}^{n}}{k_{n} / 2}\left(t-t_{n}\right) \quad \text { for } t \in I_{n},
$$

where $\bar{u}^{n}=k_{n}^{-1} \int_{t_{n-1}}^{t_{n}} u(t) d t$, and elementary calculations then show that for $t \in I_{n}$,

$$
\begin{aligned}
\Pi u(t)-u(t) & =\int_{t}^{t_{n}} u^{\prime}(s) d s-2 \frac{t_{n}-t}{k_{n}^{2}} \int_{t_{n-1}}^{t_{n}}\left(s-t_{n-1}\right) u^{\prime}(s) d s \\
& =\int_{t}^{t_{n}}\left(t_{n}-s\right) u^{\prime \prime}(s) d s+\frac{t_{n}-t}{k_{n}^{2}} \int_{t_{n-1}}^{t_{n}}\left(s-t_{n-1}\right)^{2} u^{\prime \prime}(s) d s .
\end{aligned}
$$

Using $\Pi$, we decompose the error into two terms,

$$
U-u=(U-\Pi u)+(\Pi u-u)
$$

and estimate each term separately. The representations (3.5) and (3.8) immediately yield bounds for the second term,

$$
\|\Pi u-u\|_{I_{n}} \leq C k_{n}^{r-1} \int_{t_{n-1}}^{t_{n}}\left\|u^{(r)}(t)\right\| d t \quad \text { for } 1 \leq r \leq q \leq 2,
$$

and we handle the first term as follows.

Theorem 3.1. Let $q \in\{1,2\}$. If $u$ is the solution of the initial value problem (1.1) and if $U$ is the approximate solution obtained by the discontinuous Galerkin method (1.6), then

$$
\begin{aligned}
\|U-\Pi u\|_{J_{n}} \leq C\left\|U^{0}-u_{0}\right\|+C t_{n}^{\alpha} \int_{0}^{t_{1}} t\left\|A u^{\prime}(t)\right\| d t & \\
& +C t_{n}^{\alpha} \sum_{j=2}^{n} k_{j}^{q} \int_{t_{n-1}}^{t_{n}}\left\|A u^{(q)}(t)\right\| d t .
\end{aligned}
$$

Proof. For brevity, we put

$$
\theta=U-\Pi u \text { and } \eta=\Pi u-u .
$$

The Galerkin orthogonality relation (3.3) implies that

$$
G_{N}(\theta, X)=\left\langle U^{0}-u_{0}, X_{+}^{0}\right\rangle-G_{N}(\eta, X) \text { for all } X \in \mathcal{W}_{q},
$$

and by the construction of the interpolant we have $\eta^{n}=0$ for all $n \geq 1$. Hence, using the alternative expression (3.4) for $G_{N}$,

$$
G_{N}(\eta, X)=\sum_{n=1}^{N} \int_{t_{n-1}}^{t_{n}}\left[-\left\langle\eta(t), X^{\prime}(t)\right\rangle+A(\mathcal{B} \eta(t), X(t))\right] d t .
$$

Moreover, $\int_{t_{n-1}}^{t_{n}}\left\langle\eta(t), X^{\prime}(t)\right\rangle d t=0$ if $q=1$, because $X^{\prime}(t)$ is identically zero on $I_{n}$. The same conclusion holds if $q=2$, because $X^{\prime}(t)$ is constant on $I_{n}$ and hence is orthogonal to the interpolation error. Therefore, $\theta \in \mathcal{W}_{q}$ satisfies

$$
G_{N}(\theta, X)=\left\langle U^{0}-u_{0}, X_{+}^{0}\right\rangle-\int_{0}^{T}\langle\mathcal{B} A \eta(t), X(t)\rangle d t \quad \text { for all } X \in \mathcal{W}_{q},
$$


which has the same form as the equation (3.2) satisfied by $U$, so we may apply the stability result of Theorem 2.1 and conclude that

$$
\|\theta\|_{J_{n}} \leq C\left(\left\|U^{0}-u_{0}\right\|+\int_{0}^{t_{n}}\|\mathcal{B} A \eta(t)\| d t\right) \quad \text { for } 1 \leq n \leq N .
$$

Reversing the order of integration, we find that

$$
\begin{aligned}
\int_{0}^{t_{n}}\|\mathcal{B} A \eta(t)\| d t & \leq \int_{0}^{t_{n}} \int_{0}^{t}|\beta(t, s)|\|A \eta(s)\| d s d t \\
& \leq C \int_{0}^{t_{n}} \int_{s}^{t_{n}}(t-s)^{\alpha-1} d t\|A \eta(s)\| d s \\
& =C_{\alpha} \int_{0}^{t_{n}}\left(t_{n}-s\right)^{\alpha}\|A \eta(s)\| d s \leq C_{\alpha} t_{n}^{\alpha} \int_{0}^{t_{n}}\|A \eta(s)\| d s,
\end{aligned}
$$

so

$$
\|U-\Pi u\|_{J_{n}} \leq C_{\alpha}\left(\left\|U^{0}-u_{0}\right\|+t_{n}^{\alpha} \int_{0}^{t_{n}}\|A \eta(t)\| d t\right) \quad \text { for } 1 \leq n \leq N .
$$

When $q=2$, the desired bound follows by using the formula (3.8):

$$
\begin{aligned}
\int_{0}^{t_{1}}\|A \eta(t)\| d t & \leq \int_{0}^{t_{1}}\left(\int_{t}^{t_{1}}\left\|A u^{\prime}(s)\right\| d s+2 \frac{t_{1}-t}{k_{1}^{2}} \int_{0}^{t_{1}} s\left\|A u^{\prime}(s)\right\| d s\right) d t \\
& =2 \int_{0}^{t_{1}} s\left\|A u^{\prime}(s)\right\| d s
\end{aligned}
$$

and

$$
\int_{t_{1}}^{t_{n}}\|A \eta(t)\| d t \leq \sum_{j=2}^{n} k_{j}\|A u-\Pi A u\|_{I_{j}} \leq C \sum_{j=2}^{n} k_{j}^{2} \int_{t_{j-1}}^{t_{j}}\left\|A u^{\prime \prime}(t)\right\| d t .
$$

Similar, but simpler, estimates lead to the result for $q=1$.

The next theorem shows that we can obtain $O\left(k^{q}\right)$ accuracy for all $t \in[0, T]$ provided the mesh grading, as determined by the parameter $\gamma \geq 1$, is sufficiently strong.

Theorem 3.2. Let $q \in\{1,2\}$ and assume that the step sizes are such that (1.11) and (1.12) hold. If the exact solution $u$ satisfies the regularity estimates (1.9) and (1.10), then

$$
\|U-u\|_{J_{n}} \leq C\left\|U^{0}-u_{0}\right\|+C M \times \begin{cases}k^{\gamma \sigma}, & 1 \leq \gamma<q / \sigma, \\ k^{q} \log \left(t_{n} / t_{1}\right), & \gamma=q / \sigma, \\ t_{n}^{\sigma-q / \gamma} k^{q}, & \gamma>q / \sigma .\end{cases}
$$

Proof. From (3.10), the assumptions (1.10) and (1.12) give

$$
\|\Pi u-u\|_{I_{1}} \leq C \int_{0}^{t_{1}}\left\|u^{\prime}(t)\right\| d t \leq C M \int_{0}^{t_{1}} t^{\sigma-1} d t \leq C M t_{1}^{\sigma} \leq C M k^{\gamma \sigma}
$$

and, using (1.11) for $n \geq 2$,

$$
\begin{aligned}
\|\Pi u-u\|_{I_{n}} \leq C k_{n}^{q-1} \int_{t_{n-1}}^{t_{n}}\left\|u^{(q)}(t)\right\| d t \leq & C M k_{n}^{q-1} \int_{t_{n-1}}^{t_{n}} t^{\sigma-q} d t \\
& \leq C M k_{n}^{q} t_{n}^{\sigma-q} \leq C M k^{q} t_{n}^{\sigma-q / \gamma},
\end{aligned}
$$


so we may bound the interpolation error as follows:

$$
\|\Pi u-u\|_{J_{n}}=\max _{1 \leq j \leq n}\|\Pi u-u\|_{I_{n}} \leq C M \times \begin{cases}k^{\gamma \sigma}, & 1 \leq \gamma \leq q / \sigma, \\ t_{n}^{\sigma-q / \gamma} k^{q}, & \gamma \geq q / \sigma .\end{cases}
$$

Next, by (1.9) and (1.12),

$$
\int_{0}^{t_{1}} t\left\|A u^{\prime}(t)\right\| d t \leq C M \int_{0}^{t_{1}} t^{\sigma-1} d t \leq C M k^{\gamma \sigma}
$$

and, using (1.11),

$$
\begin{aligned}
& \sum_{j=2}^{n} k_{j}^{q} \int_{t_{j-1}}^{t_{j}}\left\|A u^{(q)}(t)\right\| d t \leq C M \sum_{j=2}^{n} k_{j}^{q} \int_{t_{j-1}}^{t_{j}} t^{\sigma-1-q} d t \\
& \quad \leq C M k^{q} \sum_{j=2}^{n} t_{j}^{(1-1 / \gamma) q} \int_{t_{j-1}}^{t_{j}} t^{\sigma-1-q} d t \leq C M k^{q} \int_{t_{1}}^{t_{n}} t^{\sigma-q / \gamma-1} d t
\end{aligned}
$$

and the result follows from (3.9) and Theorem 3.1, after noting that

$$
\int_{t_{1}}^{t_{n}} t^{\sigma-q / \gamma-1} d t \leq \begin{cases}C t_{1}^{-(q / \gamma-\sigma)} \leq C k^{-(q-\gamma \sigma)}, & 1 \leq \gamma<q / \sigma \\ C \log \left(t_{n} / t_{1}\right), & \gamma=q / \sigma \\ C t_{n}^{\sigma-q / \gamma}, & \gamma>q / \sigma .\end{cases}
$$

\section{SuperconVERGENCE AT THE NODES}

We now show that for $q=2$ the numerical solution achieves a faster convergence rate at $t=t_{n}$, depending on the quantities

(4.1) $\epsilon_{n j}=\max _{t \in I_{j}}\left(\int_{t}^{t_{j}}|\beta(s, t)| d s+\int_{t_{j}}^{t_{n}}\left|\beta(s, t)-\beta\left(s, t_{j}\right)\right| d s\right) \quad$ for $1 \leq j \leq n \leq N$.

Theorem 4.1. If $u$ is the solution of the initial value problem (1.1) and if $U$ is the approximate solution obtained by the piecewise-linear $(q=2)$ discontinuous Galerkin method (1.6), then

$\left\|U^{n}-u\left(t_{n}\right)\right\| \leq C\left(\left\|U^{0}-u_{0}\right\|+\epsilon_{n 1} \int_{0}^{t_{1}} t\left\|A u^{\prime}(t)\right\| d t+\sum_{j=2}^{n} \epsilon_{n j} k_{j}^{2} \int_{t_{j-1}}^{t_{j}}\left\|A u^{\prime \prime}(t)\right\| d t\right)$.

Proof. Let $z$ be the solution of the dual problem

$$
-z^{\prime}+\mathcal{B}^{*} A z=0 \quad \text { for } 0 \leq t \leq T, \quad \text { with } z(T)=z_{T},
$$

where $\mathcal{B}^{*} v(t)=\int_{t}^{T} \beta(s, t) v(s) d s$. Since $z$ has no jumps and since

$$
\int_{0}^{T}\left[\left\langle-v(t), z^{\prime}(t)\right\rangle+A(\mathcal{B} v(t), z(t))\right] d t=\int_{0}^{T}\left\langle v(t),-z^{\prime}(t)+\mathcal{B}^{*} A z(t)\right\rangle d t=0,
$$

the formula (3.4) yields the identity

$$
G_{N}(v, z)=\left\langle v(T), z_{T}\right\rangle
$$

for all piecewise-continuous $v(t)$. Let $Z \in \mathcal{W}_{2}$ denote the approximate solution of (4.2) given by the discontinuous Galerkin method

$$
G_{N}(V, Z)=\left\langle V^{N}, z_{T}\right\rangle \quad \text { for all } V \in \mathcal{W}_{2},
$$


and let $\theta=U-\Pi u$ and $\eta=\Pi u-u$, as before, so that $U-u=\theta+\eta$. Since $u\left(t_{N}\right)=\Pi u\left(t_{N}\right)$, by taking $V=\theta$ we see from (3.3) that

$$
\left\langle U^{N}-u\left(t_{N}\right), z_{T}\right\rangle=\left\langle\theta^{N}, z_{T}\right\rangle=G_{N}(\theta, Z)=\left\langle U^{0}-u_{0}, Z_{+}^{0}\right\rangle-G_{N}(\eta, Z) .
$$

Moreover, $\eta^{n}=0$ and $\int_{t_{n-1}}^{t_{n}}\left\langle\eta(t), Z^{\prime}(t)\right\rangle d t=0$ for all $n$, so the formula (3.4) shows that

$$
G_{N}(\eta, Z)=\sum_{n=1}^{N} \delta_{n}, \quad \text { where } \quad \delta_{n}=\int_{t_{n-1}}^{t_{n}}\left\langle A \eta(t), \mathcal{B}^{*} Z(t)\right\rangle d t
$$

The orthogonality property of $\Pi$ implies that

$$
\delta_{n}=\int_{t_{n-1}}^{t_{n}}\left\langle A \eta(t), \mathcal{B}^{*} Z(t)-\mathcal{B}^{*} Z\left(t_{n}\right)\right\rangle d t,
$$

and for $t \in I_{n}$

$$
\begin{aligned}
\| \mathcal{B}^{*} Z(t) & -\mathcal{B}^{*} Z\left(t_{n}\right)\|=\| \int_{t}^{T} \beta(s, t) Z(s) d s-\int_{t_{n}}^{T} \beta\left(s, t_{n}\right) Z(s) d s \| \\
& =\left\|\int_{t}^{t_{n}} \beta(s, t) Z(s) d s+\int_{t_{n}}^{T}\left[\beta(s, t)-\beta\left(s, t_{n}\right)\right] Z(s) d s\right\| \leq \epsilon_{N n}\|Z\|_{J_{N}},
\end{aligned}
$$

so

$$
\left\|\delta_{n}\right\| \leq \epsilon_{N n}\|Z\|_{J_{N}} \int_{t_{n-1}}^{t_{n}}\|A \eta(t)\| d t .
$$

Using (3.10) we see that

$$
\int_{t_{n-1}}^{t_{n}}\|A \eta(t)\| d t \leq C k_{n}^{2} \int_{t_{n-1}}^{t_{n}}\left\|A u^{\prime \prime}(t)\right\| d t \quad \text { for } n \geq 2,
$$

and using (3.8) we find

$$
\int_{0}^{t_{1}}\|A \eta(t)\| d t \leq C \int_{0}^{t_{1}} t\left\|A u^{\prime}(t)\right\| d t .
$$

Stability of the discontinuous Galerkin method for the dual problem means that $\left\|Z_{+}^{0}\right\| \leq\|Z\|_{J_{N}} \leq C\left\|z_{T}\right\|$. Thus,

$$
\begin{aligned}
\left|\left\langle U^{N}-u\left(t_{N}\right), z_{T}\right\rangle\right| \leq C\left(\left\|U^{0}-u_{0}\right\|+\epsilon_{N 1}\right. & \int_{0}^{t_{1}} t\left\|A u^{\prime}(t)\right\| d t \\
& \left.+\sum_{n=2}^{N} \epsilon_{N n} k_{n}^{2} \int_{t_{n-1}}^{t_{n}}\left\|A u^{\prime \prime}(t)\right\| d t\right)\left\|z_{T}\right\|,
\end{aligned}
$$

and since $z_{T} \in \mathbb{H}$ is arbitrary we obtain the desired bound for $\left\|U^{N}-u\left(t_{N}\right)\right\|$.

If $\beta(t, s)$ and $u(t)$ are smooth, then $\epsilon_{n j}=O(k)$ and so $\left\|U^{n}-u\left(t_{n}\right)\right\|=O\left(k^{3}\right)$. For the specific non-smooth kernel (1.3), we have the same convergence rate provided the mesh grading is sufficiently strong.

Corollary 4.2. Let $q=2$ and $\beta(t, s)=(t-s)^{\alpha-1} / \Gamma(\alpha)$, with $0<\alpha<1$. If $u$ satisfies the regularity assumption (1.9), and if the time mesh satisfies the conditions (1.11) and (1.12), then, with $\gamma^{*}=3 /(\sigma+\alpha)$,

$$
\left\|U^{n}-u\left(t_{n}\right)\right\| \leq C\left\|U^{0}-u_{0}\right\|+C M \times \begin{cases}k^{\gamma(\sigma+\alpha)}, & 1 \leq \gamma<\gamma^{*} \\ k^{3}, & \gamma \geq \gamma^{*}\end{cases}
$$


Proof. Noting that $\beta(s, t) \geq \beta\left(s, t_{j}\right)$ for $t \leq t_{j} \leq s$, we have

$$
\begin{aligned}
& \int_{t}^{t_{j}}|\beta(s, t)| d s+\int_{t_{j}}^{t_{n}}\left|\beta(s, t)-\beta\left(s, t_{j}\right)\right| d s \\
& \quad=\int_{t}^{t_{j}} \beta(s, t) d s+\int_{t_{j}}^{t_{n}}\left(\beta(s, t)-\beta\left(s, t_{j}\right)\right) d s \\
& \quad=\int_{t}^{t_{n}} \frac{(s-t)^{\alpha-1}}{\Gamma(\alpha)} d s-\int_{t_{j}}^{t_{n}} \frac{\left(s-t_{j}\right)^{\alpha-1}}{\Gamma(\alpha)} d s=\frac{\left(t_{n}-t\right)^{\alpha}-\left(t_{n}-t_{j}\right)^{\alpha}}{\Gamma(\alpha+1)},
\end{aligned}
$$

and so $\epsilon_{n j}=\left[\left(t_{n}-t_{j-1}\right)^{\alpha}-\left(t_{n}-t_{j}\right)^{\alpha}\right] / \Gamma(\alpha+1)$. Since $X^{\alpha}-Y^{\alpha} \leq(X-Y)^{\alpha}$ for any $X \geq Y \geq 0$, we see that $\epsilon_{n j} \leq k_{j}^{\alpha} / \Gamma(\alpha+1)$, with equality when $j=n$. However, for $j<n$ we obtain a sharper bound by applying the mean value theorem: $\epsilon_{n j} \leq\left(t_{n}-t_{j}\right)^{\alpha-1} k_{j} / \Gamma(\alpha)$. Thus, using (1.9), (1.11) and (1.12), we have

$$
\epsilon_{n 1} \int_{0}^{t_{1}} t\left\|A u^{\prime}(t)\right\| d t \leq C M k_{1}^{\alpha} \int_{0}^{t_{1}} t^{\sigma-1} d t \leq C M k_{1}^{\alpha+\sigma} \leq C M k^{\gamma(\alpha+\sigma)}
$$

and, for $2 \leq j \leq n-1$,

$$
\begin{aligned}
& \epsilon_{n j} k_{j}^{2} \int_{t_{j-1}}^{t_{j}}\left\|A u^{\prime \prime}(t)\right\| d t \leq C M k_{j}^{3}\left(t_{n}-t_{j}\right)^{\alpha-1} \int_{t_{j-1}}^{t_{j}} t^{\sigma-3} d t \\
\leq & C M k^{3}\left(t_{j}\right)^{3(1-1 / \gamma)}\left(t_{n}-t_{j}\right)^{\alpha-1} \int_{t_{j-1}}^{t_{j}} t^{\sigma-3} d t \leq C M k^{3} \int_{t_{j-1}}^{t_{j}}\left(t_{n}-t\right)^{\alpha-1} t^{\sigma-3 / \gamma} d t,
\end{aligned}
$$

with

$$
\begin{aligned}
\epsilon_{n n} k_{n}^{2} \int_{t_{n-1}}^{t_{n}}\left\|A u^{\prime \prime}(t)\right\| d t & \leq C M k_{n}^{2+\alpha} \int_{t_{n-1}}^{t_{n}} t^{\sigma-3} d t \leq C M k_{n}^{3+\alpha} t_{n}^{\sigma-3} \\
& \leq C M k^{3}\left(k_{n} / t_{n}\right)^{\alpha} t_{n}^{\alpha+\sigma-3 / \gamma} \leq C M t_{n}^{\alpha+\sigma-3 / \gamma} k^{3} .
\end{aligned}
$$

Using the substitution $t=t_{n} z$, we find that

$$
\begin{aligned}
\sum_{j=2}^{n-1} \epsilon_{n j} k_{j}^{2} \int_{t_{j-1}}^{t_{j}}\left\|A u^{\prime \prime}(t)\right\| d t & \leq C M k^{3} \int_{t_{1}}^{t_{n-1}}\left(t_{n}-t\right)^{\alpha-1} t^{\sigma-3 / \gamma} d t \\
& =C M k^{3} t_{n}^{\alpha+\sigma-3 / \gamma} \int_{t_{1} / t_{n}}^{t_{n-1} / t_{n}}(1-z)^{\alpha-1} z^{\sigma-3 / \gamma} d z
\end{aligned}
$$

and an elementary calculation yields

$$
\int_{t_{1} / t_{n}}^{t_{n-1} / t_{n}}(1-z)^{\alpha-1} z^{\sigma-3 / \gamma} d z \leq C \times \begin{cases}\left(t_{1} / t_{n}\right)^{1+\sigma-3 / \gamma}, & \sigma-3 / \gamma<-1, \\ \log \left(t_{n} / t_{1}\right), & \sigma-3 / \gamma=-1, \\ 1, & \sigma-3 / \gamma>-1 .\end{cases}
$$

Theorem4.1 now shows that the nodal error $\left\|U^{n}-u\left(t_{n}\right)\right\|$ is bounded by $C\left\|U^{0}-u_{0}\right\|$ plus

$$
C M k^{\gamma(\alpha+\sigma)}+C M \times \begin{cases}t_{n}^{\alpha-1} k^{\gamma(1+\sigma)}, & 1 \leq \gamma<3 /(1+\sigma), \\ t_{n}^{\alpha-1} k^{3} \log \left(t_{n} / t_{1}\right), & \gamma=3 /(1+\sigma), \\ t_{n}^{\alpha+\sigma-3 / \gamma} k^{3}, & \gamma>3 /(1+\sigma),\end{cases}
$$

which is $O\left(k^{3}\right)$ for $\gamma \geq \gamma^{*}=3 /(\alpha+\sigma)>3 /(1+\sigma)$. If $1 \leq \gamma<3 /(1+\sigma)$, then

$$
t_{n}^{\alpha-1} k^{\gamma(1+\sigma)}=k^{\gamma(\alpha+\sigma)}\left(k^{\gamma} / t_{n}\right)^{1-\alpha} \leq C k^{\gamma(\alpha+\sigma)}\left(t_{1} / t_{n}\right)^{1-\alpha} \leq C k^{\gamma(\alpha+\sigma)} .
$$


Likewise, if $\gamma=3 /(1+\sigma)$, then

$$
\begin{aligned}
t_{n}^{\alpha-1} k^{3} \log \left(t_{n} / t_{1}\right) & =k^{\gamma(\alpha+\sigma)}\left(k^{\gamma} / t_{n}\right)^{1-\alpha} \log \left(t_{n} / t_{1}\right) \\
& \leq C k^{\gamma(\alpha+\sigma)}\left(t_{1} / t_{n}\right)^{1-\alpha} \log \left(t_{n} / t_{1}\right) \leq C k^{\gamma(\alpha+\sigma)},
\end{aligned}
$$

and in the remaining case, $3 /(1+\sigma)<\gamma<\gamma^{*}=3 /(\alpha+\sigma)$, we have

$$
t_{n}^{\alpha+\sigma-3 / \gamma} k^{3}=k^{\gamma(\alpha+\sigma)}\left(k^{\gamma} / t_{n}\right)^{3 / \gamma-(\alpha+\sigma)} \leq C k^{\gamma(\alpha+\sigma)}\left(t_{1} / t_{n}\right)^{3 / \gamma-(\alpha+\sigma)} \leq C k^{\gamma(\alpha+\sigma)} .
$$

\section{SPACE DiscretizATION}

We assume now that $\mathbb{H}=L_{2}(\Omega)$ for a bounded, convex polyhedral domain $\Omega$, and that $A$ is a strongly-elliptic, second-order, selfadjoint partial differential operator. In the case of homogeneous Dirichlet boundary conditions, we have

$$
D\left(A^{r / 2}\right)=\left\{v \in H^{r}(\Omega): v=0 \text { on } \partial \Omega\right\} \quad \text { for } 1 / 2<r \leq 2,
$$

whereas for homogeneous Neumann boundary conditions, $D\left(A^{r / 2}\right)=H^{r}(\Omega)$.

Construct a continuous, piecewise-linear finite element space $S_{h} \subseteq D\left(A^{1 / 2}\right)$ on a quasi-uniform partition of the domain $\Omega$, with $h$ denoting the maximum diameter of the elements. We then have the approximation property

$$
\min _{\chi \in S_{h}}(\|v-\chi\|+h\|\nabla(v-\chi)\|) \leq C h^{2}\|v\|_{2} \quad \text { for } v \in D(A),
$$

where we use the abbreviation $\|v\|_{r}=\|v\|_{H^{r}(\Omega)}$.

Based on the weak formulation of the initial value problem (1.1), we define a spatially-discrete, approximate solution $u_{h}:[0, T] \rightarrow S_{h}$ by requiring

$$
\left\langle u_{h}^{\prime}(t), \chi\right\rangle+\int_{0}^{t} \beta(t, s) A\left(u_{h}(s), \chi\right) d s=\langle f(t), \chi\rangle \quad \text { for } 0 \leq t \leq T \text { and all } \chi \in S_{h},
$$

with $u_{h}(0)=u_{0 h} \approx u_{0}$ for a suitable $u_{0 h} \in S_{h}$. This semi-discrete solution satisfies the error bound [12, Theorem 2.1]

$$
\left\|u_{h}(t)-u(t)\right\| \leq\left\|u_{0 h}-u_{0}\right\|+C h^{2} \int_{0}^{t}\left\|u^{\prime}(s)\right\|_{2} d s \quad \text { for } 0 \leq t \leq T .
$$

Let $\mathbb{P}_{q}\left(S_{h}\right)$ denote the space of polynomials of degree strictly less than $q$ with coefficients in $S_{h}$, and define the corresponding trial space of piecewise-polynomials $\mathcal{W}_{q}\left(S_{h}\right)$. Thus, a function $X(x, t)$ in $\mathcal{W}_{q}\left(S_{h}\right)$ is continuous in $x$ but may be discontinuous at $t=t_{n}$.

Applying the discontinuous Galerkin method in time, we arrive at a fully-discrete numerical solution $U_{h}:[0, T] \rightarrow \mathcal{W}_{q}\left(S_{h}\right)$ defined by

$$
\begin{aligned}
G_{N}\left(U_{h}, X\right) & =\left\langle U_{h}^{0}, X_{+}^{0}\right\rangle+\int_{0}^{t_{N}}\langle f(t), X(t)\rangle d t \text { for all } X \in \mathcal{W}_{q}\left(S_{h}\right), \\
U_{h}(0) & =U_{h}^{0},
\end{aligned}
$$

for a suitable $U_{h}^{0} \in S_{h}$ with $U_{h}^{0} \approx u_{0}$; cf. (3.2). In place of (3.9), we now decompose the error as

$$
U_{h}-u=\left(U_{h}-\Pi R_{h} u\right)+\left(\Pi R_{h} u-u\right),
$$


where $R_{h}: D\left(A^{1 / 2}\right) \rightarrow S_{h}$ is the Ritz projector for the (strictly) positive-definite bilinear form $A(u, v)+\langle u, v\rangle$; thus,

$$
A\left(R_{h} v, \chi\right)+\left\langle R_{h} v, \chi\right\rangle=A(v, \chi)+\langle v, \chi\rangle \quad \text { for all } \chi \in S_{h} .
$$

(The term $\langle u, v\rangle$ in the bilinear form is needed only if $A$ has a zero eigenvalue.)

Theorem 5.1. Let $q \in\{1,2\}$. If $u$ is the solution of the initial value problem (1.1), and if $U_{h} \in \mathcal{W}_{q}\left(S_{h}\right)$ is the approximate solution defined by (5.1), then

$$
\begin{array}{r}
\left\|U_{h}-\Pi R_{h} u\right\|_{J_{n}} \leq C_{T}\left(\left\|U_{h}^{0}-R_{h} u_{0}\right\|+\left\|u_{0}-R_{h} u_{0}\right\|+\int_{0}^{t_{n}}\left\|u^{\prime}(t)-R_{h} u^{\prime}(t)\right\| d t\right. \\
\left.+\int_{0}^{t_{1}} t\left\|A u^{\prime}(t)\right\| d t+\sum_{j=2}^{n} k_{j}^{q} \int_{t_{j-1}}^{t_{j}}\left\|A u^{(q)}(t)\right\| d t\right) \quad \text { for } 1 \leq n \leq N .
\end{array}
$$

Proof. The Galerkin orthogonality property (3.3) now takes the form

$$
G_{N}\left(U_{h}-u, X\right)=\left\langle U_{h}^{0}-u_{0}, X_{+}^{0}\right\rangle \text { for all } X \in \mathcal{W}_{q}\left(S_{h}\right),
$$

and for brevity we let

$$
W=\Pi R_{h} u \quad \text { and } \quad \xi=R_{h} u-u .
$$

Adapting the proof of Theorem 3.1, we see from (5.4) that

$$
G_{N}\left(U_{h}-W, X\right)=\left\langle U_{h}^{0}-u_{0}, X_{+}^{0}\right\rangle-G_{N}(W-u, X) \text { for all } X \in \mathcal{W}_{q}\left(S_{h}\right),
$$

and, because $W^{n}=R_{h} u\left(t_{n}\right)$, the formula (3.4) gives

$$
\begin{aligned}
G_{N}(W-u, X)=\left\langle\xi^{N}, X^{N}\right\rangle & -\sum_{n=1}^{N-1}\left\langle\xi^{n},[X]^{n}\right\rangle \\
& +\sum_{n=1}^{N} \int_{t_{n-1}}^{t_{n}}\left[-\left\langle W-u, X^{\prime}\right\rangle+A(\mathcal{B}(W-u), X)\right] d t .
\end{aligned}
$$

Since $\int_{t_{n-1}}^{t_{n}}\left\langle W-R_{h} u, X^{\prime}\right\rangle d t=\int_{t_{n-1}}^{t_{n}}\left\langle\Pi\left(R_{h} u\right)-\left(R_{h} u\right), X^{\prime}\right\rangle d t=0$, an integration by parts shows that

$$
\begin{aligned}
\int_{t_{n-1}}^{t_{n}}-\left\langle W-u, X^{\prime}\right\rangle d t & =\int_{t_{n-1}}^{t_{n}}-\left\langle R_{h} u-u, X^{\prime}\right\rangle d t=\int_{t_{n-1}}^{t_{n}}-\left\langle\xi, X^{\prime}\right\rangle d t \\
& =-\left\langle\xi^{n}, X^{n}\right\rangle+\left\langle\xi^{n-1}, X_{+}^{n-1}\right\rangle+\int_{t_{n-1}}^{t_{n}}\left\langle\xi^{\prime}, X\right\rangle d t,
\end{aligned}
$$

and, with $\eta=\Pi u-u$, the definition of the Ritz projector gives

$$
\begin{aligned}
A((W-u)(s), X(t)) & =A\left(R_{h} \Pi u(s)-u(s), X(t)\right) \\
& =A((\Pi u-u)(s), X(t))+\left\langle\Pi\left(u-R_{h} u\right)(s), X(t)\right\rangle \\
& =\langle A \eta(s)-\Pi \xi(s), X(t)\rangle,
\end{aligned}
$$

so

$$
G_{N}(W-u, X)=\left\langle\xi^{0}, X_{+}^{0}\right\rangle+\int_{0}^{t_{N}}\left\langle\xi^{\prime}+\mathcal{B} A \eta-\mathcal{B} \Pi \xi, X\right\rangle d t .
$$

Thus, from (5.5),

$$
G_{N}\left(U_{h}-W, X\right)=\left\langle U_{h}^{0}-R_{h} u_{0}, X_{+}^{0}\right\rangle-\int_{0}^{t_{N}}\left\langle\xi^{\prime}+\mathcal{B} A \eta-\mathcal{B} \Pi \xi, X\right\rangle d t
$$


for all $X \in \mathcal{W}_{q}\left(S_{h}\right)$; cf. (3.11). Stability of the discontinuous Galerkin method (Theorem 2.1 with $\mathbb{H}=S_{h}$ ) now yields the estimate

$$
\left\|U_{h}-W\right\|_{J_{n}} \leq C\left(\left\|U_{h}^{0}-R_{h} u_{0}\right\|+\int_{0}^{t_{n}}\left\|\xi^{\prime}+\mathcal{B} A \eta-\mathcal{B} \Pi \xi\right\| d t\right) .
$$

We already estimated the term $\int_{0}^{t_{n}}\|\mathcal{B} A \eta\| d t$ in Theorem 3.1 and, for the remaining terms in the integral, we apply the bound $\|\Pi v\|_{I_{n}} \leq C\|v\|_{I_{n}}$ and arrive at

$$
\int_{0}^{t_{n}}\left\|\xi^{\prime}(t)-\mathcal{B} \Pi \xi\right\| d t \leq \int_{0}^{t_{n}}\left\|\xi^{\prime}\right\| d t+C t_{n}^{\alpha+1}\|\xi\|_{J_{n}} \leq C_{T}\left(\|\xi(0)\|+\int_{0}^{t_{n}}\left\|\xi^{\prime}\right\| d t\right) .
$$

We can now show that the space discretisation leads to an additional error of order $h^{2} \ell(k)$ compared with the error bound of Theorem 3.2, recall from (1.15) that $\ell(k)=\max \left(1, \log k^{-1}\right)$.

Theorem 5.2. Let $q \in\{1,2\}$ and assume that the time mesh is such that (1.11) and (1.12) hold. If the exact solution $u$ satisfies the regularity estimates (1.9), (1.10) and (1.14), then, for $1 \leq n \leq N$ and with $C=C_{T}$,

$$
\left\|U_{h}-u\right\|_{J_{n}} \leq C\left\|U_{h}^{0}-u_{0}\right\|+C M h^{2} \ell\left(t_{n} / t_{1}\right)+C M \times \begin{cases}k^{\gamma \sigma}, & 1 \leq \gamma<q / \sigma \\ k^{q} \ell\left(t_{n} / t_{1}\right), & \gamma=q / \sigma \\ k^{q}, & \gamma>q / \sigma .\end{cases}
$$

Proof. Recall that

$$
\left\|v-R_{h} v\right\| \leq C h^{2}\|v\|_{2} .
$$

To estimate the second term $\Pi R_{h} u-u$ in the decomposition (5.2), we again write $\xi=R_{h} u-u$ and then use $\|\Pi v\|_{J_{n}} \leq C\|v\|_{J_{n}}$ to obtain

$$
\left\|\Pi R_{h} u-u\right\|_{J_{n}}=\|\Pi u-u+\Pi \xi\|_{J_{n}} \leq\|\Pi u-u\|_{J_{n}}+C\|\xi(0)\|+C \int_{0}^{t_{n}}\left\|\xi^{\prime}\right\| d t .
$$

In view of Theorems 3.2 and [5.1, and the fact that $\left\|U_{h}^{0}-R_{h} u_{0}\right\| \leq\left\|U_{h}^{0}-u_{0}\right\|+$ $\left\|u_{0}-R_{h} u_{0}\right\|$, it suffices to note that $\|\xi(0)\| \leq C h^{2}\left\|u_{0}\right\|_{2}$ and, using (1.10), (1.11), (1.12) and (1.14),

$$
\begin{aligned}
\int_{0}^{t_{n}}\left\|\xi^{\prime}(t)\right\| d t & \leq C \int_{0}^{t_{1}}\left\|u^{\prime}(t)\right\| d t+\int_{t_{1}}^{t_{n}} C h^{2}\left\|u^{\prime}(t)\right\|_{2} d t \\
& \leq C M \int_{0}^{t_{1}} t^{\sigma-1} d t+C M h^{2} \int_{t_{1}}^{t_{n}} t^{-1} d t \\
& \leq C M k^{\gamma \sigma}+C M h^{2} \ell\left(t_{n} / t_{1}\right) .
\end{aligned}
$$

Next, we prove a spatially-discrete version of Theorem 4.1 showing superconvergence at $t=t_{n}$.

Theorem 5.3. Let $q=2$ and define $\epsilon_{n j}$ by (4.1). If $u$ is the solution of (1.1) and if $U_{h} \in \mathcal{W}_{2}\left(S_{h}\right)$ is the approximate solution given by (5.1), then

$$
\begin{aligned}
\left\|U_{h}^{n}-u\left(t_{n}\right)\right\| \leq C_{T}\left(\left\|U_{h}^{0}-u_{0}\right\|+\left\|u_{0}-R_{h} u_{0}\right\|+\int_{0}^{t_{n}}\left\|u^{\prime}(t)-R_{h} u^{\prime}(t)\right\| d t\right. \\
\left.\quad+\epsilon_{n 1} \int_{0}^{t_{1}} t\left\|A u^{\prime}(t)\right\| d t+\sum_{j=2}^{n} \epsilon_{n j} k_{j}^{2} \int_{t_{n-1}}^{t_{n}}\left\|A u^{\prime \prime}(t)\right\| d t\right) \quad \text { for } 1 \leq n \leq N .
\end{aligned}
$$


Proof. We adapt the proof of Theorem 4.1, letting $Z \in \mathcal{W}_{2}\left(S_{h}\right)$ denote the solution of

$$
G_{N}(V, Z)=\left\langle V^{N}, z_{T}\right\rangle \quad \text { for all } V \in \mathcal{W}_{2}\left(S_{h}\right)
$$

and writing

$$
W=\Pi R_{h} u, \quad \eta=\Pi u-u, \quad \xi=R_{h} u-u .
$$

The Galerkin orthogonality (5.4) implies, cf. (4.3),

$$
\begin{aligned}
\left\langle U_{h}^{N}-R_{h} u\left(t_{N}\right), z_{T}\right\rangle & =\left\langle U_{h}^{N}-W^{N}, z_{T}\right\rangle=G_{N}\left(U_{h}-W, Z\right) \\
& =G_{N}\left(U_{h}-u, Z\right)+G_{N}(u-W, Z) \\
& =\left\langle U_{h}^{0}-u_{0}, Z_{+}^{0}\right\rangle-G_{N}(\eta, Z)-G_{N}(\Pi \xi, Z),
\end{aligned}
$$

and by the triangle inequality,

$$
\left\|U_{h}^{N}-u\left(t_{N}\right)\right\| \leq\left\|U_{h}^{N}-R_{h} u\left(t_{N}\right)\right\|+\|\xi(0)\|+\int_{0}^{t_{N}}\left\|\xi^{\prime}(t)\right\| d t,
$$

so it suffices to prove that

$$
\left|G_{N}(\Pi \xi, Z)\right| \leq C_{T}\left\|z_{T}\right\|\left(\|\xi(0)\|+\int_{0}^{t_{N}}\left\|\xi^{\prime}(t)\right\| d t\right) .
$$

From the definition (3.1) of $G_{N}$,

$$
\begin{aligned}
G_{N}(\Pi \xi, Z)=\left\langle\Pi \xi_{+}^{0}, Z_{+}^{0}\right\rangle+ & \sum_{n=1}^{N-1}\left\langle[\Pi \xi]^{n}, Z_{+}^{n}\right\rangle \\
& +\sum_{n=1}^{N} \int_{t_{n-1}}^{t_{n}}\left[\left\langle(\Pi \xi)^{\prime}(t), Z(t)\right\rangle+A(\mathcal{B} \Pi \xi(t), Z(t))\right] d t,
\end{aligned}
$$

and from the definition (5.3) of $R_{h}$,

$$
A(\mathcal{B} \Pi \xi(t), Z(t))=-\langle\mathcal{B} \Pi \xi(t), Z(t)\rangle .
$$

Integrating by parts, applying the orthogonality and interpolation propertes of $\Pi$ and noting that $\xi_{+}^{n-1}=\xi\left(t_{n-1}\right)=(\Pi \xi)^{n-1}$, we have

$$
\begin{aligned}
\int_{t_{n-1}}^{t_{n}}\left\langle(\Pi \xi)^{\prime}(t), Z(t)\right\rangle d t & =\left\langle(\Pi \xi)^{n}, Z^{n}\right\rangle-\left\langle(\Pi \xi)_{+}^{n-1}, Z_{+}^{n-1}\right\rangle-\int_{t_{n-1}}^{t_{n}}\left\langle\Pi \xi(t), Z^{\prime}(t)\right\rangle d t \\
& =\left\langle\xi^{n}, Z^{n}\right\rangle-\left\langle(\Pi \xi)_{+}^{n-1}, Z_{+}^{n-1}\right\rangle-\int_{t_{n-1}}^{t_{n}}\left\langle\xi(t), Z^{\prime}(t)\right\rangle d t \\
& =\left\langle\xi_{+}^{n-1}-(\Pi \xi)_{+}^{n-1}, Z_{+}^{n-1}\right\rangle+\int_{t_{n-1}}^{t_{n}}\left\langle\xi^{\prime}(t), Z(t)\right\rangle d t \\
& =-\left\langle[\Pi \xi]^{n-1}, Z_{+}^{n-1}\right\rangle+\int_{t_{n-1}}^{t_{n}}\left\langle\xi^{\prime}(t), Z(t)\right\rangle d t .
\end{aligned}
$$

Thus,

$$
G_{N}(\Pi \xi, Z)=\left\langle\xi(0), Z_{+}^{0}\right\rangle+\int_{t_{n-1}}^{t_{n}}\left\langle\xi^{\prime}(t)-\mathcal{B} \Pi \xi(t), Z(t)\right\rangle d t,
$$

and hence, noting that $\|Z\|_{J_{N}} \leq C\left\|z_{T}\right\|$ by stability of the dual problem,

$$
\left|G_{N}(\Pi \xi, Z)\right| \leq C\left\|z_{T}\right\|\left(\|\xi(0)\|+\int_{0}^{t_{N}}\left[\left\|\xi^{\prime}(t)\right\|+\|\mathcal{B} \Pi \xi(t)\|\right] d t\right) .
$$


The representation (3.7) implies that $\|\Pi u\|_{I_{n}} \leq C\|u\|_{I_{n}}$. Thus,

$$
\int_{0}^{t_{N}}\|\mathcal{B} \Pi \xi(t)\| d t \leq \int_{0}^{t_{N}} \int_{0}^{t}|\beta(t, s)|\|\Pi \xi(s)\| d s d t \leq C_{T}\|\xi\|_{J_{N}}
$$

so (5.6) follows using the bound $\|\xi\|_{J_{N}} \leq\|\xi(0)\|+\int_{0}^{t_{N}}\left\|\xi^{\prime}(t)\right\| d t$.

Corollary 5.4. Let $q=2$, assume $\beta$ is the weakly singular kernel (1.3) and suppose that the time mesh satisfies (1.11) and (1.12). If the regularity estimates (1.9), (1.10) and (1.14) hold, then, with $\gamma^{*}=3 /(\sigma+\alpha)$ and $C=C_{T}$,

$$
\left\|U_{h}^{n}-u\left(t_{n}\right)\right\| \leq C\left\|U_{h}^{0}-u_{0}\right\|+C M h^{2} \ell(k)+C M \times \begin{cases}k^{\gamma(\sigma+\alpha)}, & 1 \leq \gamma<\gamma^{*}, \\ k^{3}, & \gamma \geq \gamma^{*}\end{cases}
$$

for $1 \leq n \leq N$.

Proof. Use Theorem 5.3 and apply the estimates from the proofs of Theorem 5.2 and Corollary 4.2 .

\section{NumERICAL EXPERIMENTS}

We now apply the discontinuous Galerkin method (1.6) and its spatially-discrete version (5.1) to some problems of the form (1.1). In each case the time interval is $[0, T]=[0,1]$ and we employ a time mesh of the form (1.13) for various choices of the mesh grading parameter $\gamma \geq 1$. We consider only the piecewise-linear case $q=2$.

6.1. Scalar examples. To demonstrate the effect of the time discretization by itself, with no additional errors arising from a spatial discretization, we first consider a purely time-dependent problem

$$
\frac{d u}{d t}+\int_{0}^{t} \beta(t-s) u(s) d s=f(t) \quad \text { for } 0<t<T \text { with } u(0)=u_{0},
$$

with the weakly singular kernel $\beta(t)=t^{\alpha-1} / \Gamma(\alpha)$ for $0<\alpha<1$. Using the MittagLeffler function $E_{\mu}(x)=\sum_{p=0}^{\infty} x^{p} / \Gamma(1+p \mu)$, we may write the exact solution as

$$
u(t)=E_{\alpha+1}\left(-t^{\alpha+1}\right) u_{0}+\int_{0}^{t} E_{\alpha+1}\left(-s^{\alpha+1}\right) f(t-s) d s
$$

see [11. Choosing initial data $u_{0}=0$ and a source term $f(t)=(\alpha+1) t^{\alpha}$, we find that

$$
u(t)=-\Gamma(\alpha+2) \sum_{p=1}^{\infty} \frac{(-t)^{(\alpha+1) p}}{\Gamma(1+(\alpha+1) p)}=\Gamma(\alpha+2)\left(1-E_{\alpha+1}\left(-t^{\alpha+1}\right)\right) .
$$

To tabulate our numerical results, we introduce a finer grid

$$
\mathcal{G}^{N, m}=\left\{t_{j-1}+\ell k_{j} / m: j=1,2, \ldots, N \text { and } \ell=0,1, \ldots, m\right\}
$$

and an associated norm $\|v\|_{\infty}^{N, m}=\max _{t \in \mathcal{G}^{N, m}}|v(t)|$. Thus, $\|U-u\|_{\infty}^{N, 1}$ is the maximum error at the nodes whereas, for larger values of $m$, the norm $\|U-u\|_{\infty}^{N, m}$ approximates the uniform error $\|U-u\|_{L_{\infty}(0, T)}$.

Since the exact solution (6.1) behaves like $t^{\alpha+1}$ as $t \rightarrow 0^{+}$, we see that the first regularity condition (1.9) holds for any $\sigma \leq \alpha+2$ and the second condition (1.10) holds for any $\sigma \leq \alpha+1$. Thus, from Theorem 3.2 we expect $\|U-u\|_{J_{n}}$ to be $O\left(k^{\gamma \sigma}\right)$ for $1 \leq \gamma<2 /(\alpha+1)$, and $O\left(k^{2}\right)$ for $\gamma>2 /(\alpha+1)$. Results for $\alpha=0.4$, shown in Table 1, are consistent with these error bounds. 
TABLE 1. The error $\|U-u\|_{\infty}^{N, 5}$ with different mesh gradings, when $\alpha=0.4$. We observe $O\left(k^{(\alpha+1) \gamma}\right)$ convergence if $1 \leq \gamma<2 /(\alpha+1) \approx$ 1.4286, and $O\left(k^{2}\right)$ convergence if $\gamma>2 /(\alpha+1)$.

\begin{tabular}{|r|cc|cc|cc|cc|}
\hline$N$ & \multicolumn{2}{|c|}{$\gamma=1$} & \multicolumn{2}{c|}{$\gamma=1.25$} & \multicolumn{2}{c|}{$\gamma=1.45$} & \multicolumn{2}{c|}{$\gamma=2$} \\
\hline 40 & $2.26 \mathrm{e}-04$ & & $6.25 \mathrm{e}-05$ & & $3.38 \mathrm{e}-05$ & & $4.27 \mathrm{e}-05$ & \\
80 & $8.61 \mathrm{e}-05$ & 1.39 & $1.86 \mathrm{e}-05$ & 1.74 & $8.59 \mathrm{e}-06$ & 1.97 & $1.09 \mathrm{e}-05$ & 1.96 \\
160 & $3.26 \mathrm{e}-05$ & 1.39 & $5.53 \mathrm{e}-06$ & 1.74 & $2.16 \mathrm{e}-06$ & 1.98 & $2.77 \mathrm{e}-06$ & 1.98 \\
320 & $1.23 \mathrm{e}-05$ & 1.39 & $1.64 \mathrm{e}-06$ & 1.74 & $5.55 \mathrm{e}-07$ & 1.99 & $6.96 \mathrm{e}-07$ & 1.99 \\
640 & $4.69 \mathrm{e}-06$ & 1.39 & $4.89 \mathrm{e}-07$ & 1.74 & $1.36 \mathrm{e}-07$ & 1.99 & $1.74 \mathrm{e}-07$ & 1.99 \\
\hline
\end{tabular}

TABLE 2. The nodal error $\|U-u\|_{\infty}^{N, 1}$ with different mesh gradings, when $\alpha=0.2$. We observe $O\left(k^{\gamma(2 \alpha+2)}\right)$ convergence for $1 \leq \gamma<$ $\gamma^{*}=3 /(2 \alpha+2)=1.25$, and $O\left(k^{3}\right)$ convergence for $\gamma \geq \gamma^{*}$.

\begin{tabular}{|r|cc|cc|cc|}
\hline$N$ & \multicolumn{2}{|c|}{$\gamma=1$} & \multicolumn{2}{c|}{$\gamma=1.25$} & \multicolumn{2}{c|}{$\gamma=1.5$} \\
\hline 40 & $2.73 \mathrm{e}-07$ & & $6.34 \mathrm{e}-08$ & & $9.51 \mathrm{e}-08$ & \\
80 & $5.37 \mathrm{e}-08$ & 1.34 & $9.06 \mathrm{e}-09$ & 2.80 & $1.38 \mathrm{e}-08$ & 2.78 \\
160 & $1.03 \mathrm{e}-08$ & 1.37 & $1.25 \mathrm{e}-09$ & 2.85 & $1.94 \mathrm{e}-09$ & 2.84 \\
320 & $1.97 \mathrm{e}-09$ & 2.39 & $1.69 \mathrm{e}-10$ & 2.88 & $2.65 \mathrm{e}-10$ & 2.87 \\
640 & $3.74 \mathrm{e}-10$ & 2.39 & $2.26 \mathrm{e}-11$ & 2.90 & $3.57 \mathrm{e}-11$ & 2.90 \\
1280 & $7.11 \mathrm{e}-11$ & 2.39 & $2.98 \mathrm{e}-12$ & 2.93 & $4.73 \mathrm{e}-12$ & 2.92 \\
\hline
\end{tabular}

In Corollary 4.2 we may take $\sigma=\alpha+2$, leading to $\gamma^{*}=3 /(2 \alpha+2)$ and an expected nodal error of order $k^{3}$ for any $\gamma \geq \gamma^{*}$. This predicted behaviour is consistent with the numerical results in Table 2, where $\alpha=0.2$.

We also consider an example with the smooth kernel $\beta(t)=e^{-2 t}$. The exact solution has the form

$$
u(t)=W(t) u_{0}+\int_{0}^{t} W(t-s) f(s) d s, \quad \text { where } \quad W(t)=(1+t) e^{-t},
$$

see [12, Section 6], so for the particular choices $u_{0}=1$ and $f(t)=t e^{t}$ we have

(6.3) $u(t)=(1+t) e^{-t}+\int_{0}^{t}(1+s) e^{-s}(t-s) e^{t-s} d s=\frac{3 t}{2} \cosh t-\sinh t+(1+t / 2) e^{-t}$.

Table 3 shows that, for a uniform mesh, we obtain $O\left(k^{2}\right)$ convergence globally and $O\left(k^{3}\right)$ convergence at the nodes, as expected from the error bounds in Theorems 3.1 and 4.1 .

\subsection{A problem in one space dimension. Let}

$$
\beta(t)=\frac{t^{\alpha-1}}{\Gamma(\alpha)}, \quad \Omega=(0,1), \quad A u=-u_{x x},
$$

and assume that $u=u(x, t)$ satisfies the homogeneous Dirichlet boundary conditions $u(0, t)=0=u(1, t)$ for all $t \in[0, T]=[0,1]$. The solution operator for the 
TABLE 3. Global and nodal errors for a uniform mesh $(\gamma=1)$ when $\beta(t)=e^{-2 t}$. We observe $O\left(k^{2}\right)$ and $O\left(k^{3}\right)$ convergence, respectively.

\begin{tabular}{|r|cc|cc|}
\hline$N$ & \multicolumn{2}{|c|}{$\|U-u\|_{\infty}^{N, 5}$} & \multicolumn{2}{|c|}{$\|U-u\|_{\infty}^{N, 1}$} \\
\hline 40 & $1.56 \mathrm{e}-04$ & & $2.55 \mathrm{e}-07$ & \\
80 & $3.92 \mathrm{e}-05$ & 1.991 & $3.20 \mathrm{e}-08$ & 2.998 \\
160 & $9.83 \mathrm{e}-06$ & 1.995 & $4.00 \mathrm{e}-09$ & 2.999 \\
320 & $2.46 \mathrm{e}-06$ & 1.997 & $5.00 \mathrm{e}-10$ & 2.999 \\
640 & $6.16 \mathrm{e}-07$ & 1.998 & $6.25 \mathrm{e}-11$ & 3.000 \\
\hline
\end{tabular}

homogeneous problem $(f \equiv 0)$ is given in terms of the Mittag-Leffler function and the eigensystem of $A$ by

$$
\mathcal{E}(t) v=\sum_{m=1}^{\infty}\left\langle v, \phi_{m}\right\rangle E_{\alpha+1}\left(-\lambda_{m} t^{\alpha+1}\right) \phi_{m}, \quad \lambda_{m}=(m \pi)^{2}, \quad \phi_{m}(x)=\sqrt{2} \sin (m \pi x),
$$

and for the inhomogeneous problem a Duhamel principle yields an integral representation

$$
u(t)=\mathcal{E}(t) u_{0}+\int_{0}^{t} \mathcal{E}(t-s) f(s) d s
$$

see [11] or [12]. We choose $u_{0}(x)=\phi_{1}(x) / \sqrt{2}=\sin (\pi x)$ for the initial data and $f(t, x)=(\alpha+1) t^{\alpha} \sin (\pi x)$ for the inhomogeneous term, and we find that

$$
u(t)=\left\{E_{\alpha+1}\left(-\pi^{2} t^{\alpha+1}\right)\left(1-\frac{\Gamma(\alpha+2)}{\pi^{2}}\right)+\frac{\Gamma(\alpha+2)}{\pi^{2}}\right\} \sin (\pi x) .
$$

Thus, the first regularity condition (1.9) holds for $\sigma \leq \alpha+2$, the second condition (1.10) holds for $\sigma \leq \alpha+1$, and the additional assumption (1.14) is also satisfied.

We apply our fully discrete scheme (5.1) with a time mesh of the form (1.13) and a uniform spatial mesh with $N_{x}$ subintervals, each of length $h=1 / N_{x}$. We choose $U_{h}^{0}$ to be the $L_{2}$ projection of the initial data $u_{0}$ onto the space of continuous, piecewise-linear functions $S_{h}$. Taking $\sigma=\alpha+1$ in Theorem 5.2 we see that the global error $\left\|U_{h}-u\right\|_{L_{\infty}\left(L_{2}\right)}$ is of order $h^{2} \ell(k)+k^{\gamma(\alpha+1)}$ for $1 \leq \gamma<2 /(\alpha+1)$, and of order $h^{2} \ell(k)+k^{2}$ for $\gamma>2 /(\alpha+1)$. With $\alpha=0.4$ and defining the norm $\|v\|_{\infty}^{N, m}=\max _{t \in \mathcal{G}^{N, m}}\|v\|_{L_{2}(\Omega)}$, we obtain the results shown in Table 4. which are consistent with our theoretical error bounds. Putting $\sigma=\alpha+2$ in Corollary 5.4 gives $\gamma^{*}=3 /(2 \alpha+2)$, so we expect the nodal error $\left\|U_{h}\left(t_{n}\right)-u\left(t_{n}\right)\right\|$ to be of order $h^{2} \ell(k)+k^{\gamma(2 \alpha+2) \gamma}$ for $1 \leq \gamma<\gamma^{*}$ and of order $h^{2} \ell(k)+k^{3}$ for $\gamma \geq \gamma^{*}$. We observe this behaviour in Table 5 for the case $\alpha=0.3$. 
TABLE 4. The error $\left\|U_{h}-u\right\|_{\infty}^{N, 5}$ with different mesh gradings, when $\alpha=0.4$. Taking $N_{x}=N$, we observe convergence of order $h^{2} \ell(k)+k^{(\alpha+1) \gamma}$ for $1 \leq \gamma \leq 2 /(\alpha+1) \approx 1.4286$, and of order $h^{2} \ell(k)+k^{2}$ for $\gamma>2 /(\alpha+1)$.

\begin{tabular}{|r|cc|cc|cc|}
\hline$N=N^{x}$ & \multicolumn{2}{|c|}{$\gamma=1$} & \multicolumn{2}{c|}{$\gamma=1.45$} & \multicolumn{2}{c|}{$\gamma=2$} \\
\hline 20 & $2.69 \mathrm{e}-03$ & & $1.26 \mathrm{e}-03$ & & $1.38 \mathrm{e}-03$ & \\
40 & $1.07 \mathrm{e}-03$ & 1.31 & $3.23 \mathrm{e}-04$ & 1.96 & $3.54 \mathrm{e}-04$ & 1.96 \\
80 & $4.17 \mathrm{e}-04$ & 1.36 & $8.15 \mathrm{e}-05$ & 1.98 & $8.97 \mathrm{e}-05$ & 1.98 \\
160 & $1.59 \mathrm{e}-04$ & 1.38 & $2.04 \mathrm{e}-05$ & 1.99 & $2.25 \mathrm{e}-05$ & 1.99 \\
320 & $6.07 \mathrm{e}-05$ & 1.39 & $5.12 \mathrm{e}-06$ & 1.99 & $5.65 \mathrm{e}-06$ & 1.99 \\
\hline
\end{tabular}

TABLE 5. The nodal error $\left\|U_{h}-u\right\|_{\infty}^{N, 1}$ for various mesh gradings, when $\alpha=0.3$. We observe convergence of order $h^{2} \ell(k)+k^{\gamma(2 \alpha+1)}$ for $1 \leq \gamma<\gamma^{*}=3 /(2 \alpha+2) \approx 1.1538$, and of order $h^{2} \ell(k)+k^{3}$ for $\gamma \geq \gamma^{*}$.

\begin{tabular}{|r|cc|cc|cc|cc|}
\hline & \multicolumn{2}{|c|}{$N^{x}=N$} & \multicolumn{5}{c|}{$N^{x}=N^{3 / 2}$} \\
\hline$N$ & \multicolumn{2}{|c|}{$\gamma=1$} & $\gamma=1$ & \multicolumn{2}{c|}{$\gamma=1.2$} & \multicolumn{2}{c|}{$\gamma=1.4$} \\
\hline 36 & $2.25 \mathrm{e}-04$ & $1.67 \mathrm{e}-05$ & & $6.67 \mathrm{e}-06$ & & $6.14 \mathrm{e}-06$ & \\
49 & $1.22 \mathrm{e}-04$ & 1.98 & $8.03 \mathrm{e}-06$ & 2.37 & $2.71 \mathrm{e}-06$ & 2.91 & $2.40 \mathrm{e}-06$ & 3.03 \\
64 & $7.23 \mathrm{e}-05$ & 1.98 & $4.18 \mathrm{e}-06$ & 2.44 & $1.25 \mathrm{e}-06$ & 2.90 & $1.07 \mathrm{e}-06$ & 3.03 \\
81 & $4.53 \mathrm{e}-05$ & 1.96 & $2.33 \mathrm{e}-06$ & 2.48 & $6.30 \mathrm{e}-07$ & 2.91 & $5.25 \mathrm{e}-07$ & 3.02 \\
100 & $2.98 \mathrm{e}-05$ & 2.00 & $1.37 \mathrm{e}-06$ & 2.51 & $3.40 \mathrm{e}-07$ & 2.91 & $2.77 \mathrm{e}-07$ & 3.02 \\
121 & $2.04 \mathrm{e}-05$ & 1.99 & $8.34 \mathrm{e}-07$ & 2.61 & $1.95 \mathrm{e}-07$ & 2.92 & $1.56 \mathrm{e}-07$ & 3.02 \\
\hline
\end{tabular}

\section{REFERENCES}

1. K. Adolfsson, M. Enelund and S. Larsson, Adaptive discretization of an integro-differential equation with a weakly singluar kernel, Comput. Methods Appl. Mech. Engrg., 192, 5285-5304 (2003). MR2023899 (2004j:65218)

2. B. Cockburn, G. E. Karniadakis and C.-W. Shu (Eds.), Discontinuous Galerkin Methods: Theory, Computation and Algorithms, Lecture Notes in Computational Science and Engineering 11, Springer 2000. MR.1842160 (2002b:65004)

3. E. Cuesta, C. H. Lubich and C. Palencia, Convolution quadrature time discretization of fractional diffusion-wave equations, Math. Comp., 75, 673-696 (2006). MR2196986|(2006j:65404)

4. K. Eriksson and C. Johnson, Adaptive finite element methods for parabolic problems. I. A linear model problem, SIAM J. Numer. Anal., 28, 199-208 (1991). MR.1083324 (91m:65274)

5. K. Eriksson, C. Johnson and Thomée, Time discretization of parabolic problems by the discontinuous Galerkin method, RAIRO Modél. Math. Anal. Numér., 19, 611-643 (1985). MR 826227 (87e:65073)

6. S. Larsson, V. Thomée and L. B. Wahlbin, Numerical solution of parabolic integro-differential equations by the discontinuous Galerkin method, Math. Comp., 67, 45-71 (1998). MR.1432129 (98d:65168)

7. M. López-Fernández and C. Palencia, On the numerical inversion of the Laplace transform of certain holomorphic functions, Appl. Numer. Math., 51, 289-303 (2004). MR2091405 (2005e:65210) 
8. M. López-Fernandez, C. Palencia and A. Schädle, A spectral order method for inverting sectorial Laplace transforms, SIAM J. Numer. Anal. 44, 1332-1350 (2006). MR 2231867 (2007e:65138)

9. J.-C. López Marcos, A difference scheme for a nonlinear partial integrodifferential equation, SIAM J. Numer. Anal., 27, 20-31 (1990). MR.1034918 (91e:65160)

10. C. H. Lubich, I. H. Sloan and V. Thomée, Nonsmooth data error estimates for approximations of an evolution equation with a positive-type memory term, Math. Comp., 65, 1-17 (1996). MR.1322891 (96d:65207)

11. W. McLean and K. Mustapha, A second-order accurate numerical method for a fractional wave equation, Numer. Math., 105, 481-510 (2007). MR.2266834 (2008d:65097)

12. W. McLean and V. Thomée, Numerical solution of an evolution equation with a positive-type memory term, J. Austral. Math. Soc. Ser. B, 35, 23-70 (1993). MR.1225703 (94e:65094)

13. W. McLean and V. Thomée, Time discretization of an evolution equation via Laplace transforms, IMA. J. Numer. Anal., 24, 439-463 (2004). MR2068831 (2005d:47072)

14. W. McLean and V. Thomée, Numerical solution via Laplace transforms of a fractional order evolution equation, J. Integral Equations Appl., to appear.

15. W. McLean, V. Thomée and L. B. Wahlbin, Discretization with variable time steps of an evolution equation with a positive-type memory term, J. Comput. Appl. Math., 69, 49-69 (1996). MR:1391611 (97b:65073)

16. M. J. Sanz-Serna, A numerical method for a partial integro-differential equation, SIAM J. Numer. Anal., 25, 319-327 (1988). MR.933727 (89d:65113)

17. Achim Schädle, María López-Fernández and Christian Lubich, Fast and oblivious convolution quadrature, SIAM J. Sci. Comput., 28, 421-438 (2006). MR2231714(2007b:65142)

18. W. R. Schneider and W. Wyss, Fractional diffusion and wave equations, J. Math. Phys., 30, 134-144 (1989). MR974464 (89m:45017)

Department of Mathematics and Statistics, King Fahd University of Petroleum and Minerals, Dhahran, 31261, Saudi Arabia

E-mail address: kassem@kfupm.edu.sa

School of Mathematics and Statistics, The University of New South Wales, Sydney 2052, Australia

E-mail address: w.mclean@unsw.edu.au 